\title{
Effect of Cyberknife Radiosurgery for Elderly Patients with Trigeminal Neuralgia
}

Dong-Eon Lee, Min-Soo Kim, Jong-Ho Ahn, Seu-Ryang Jang, Eun-Hye Lee, Min-Ho Lee, Jae-Jeon Shin, Sang-Bok Lee, Young-Woo Kim, Chul Ji, Tae-Kyu Lee

Department of Neurosurgery, Uijeongbu St. Mary's Hospital, The Catholic University of Korea, College of Medicine, Uijeongbu, Republic of Korea

\begin{abstract}
Objective: We retrospectively analyzed the outcome of 28 patients over 65 years old with medically intractable Trigeminal Neuralgia (TN) to evaluate whether CyberKnife RadioSurgery (CKRS) is an effective and safe modality of therapy. Methods: We collect retrospective data on 28 patients undergoing CKRS in patients with TN since April 1, 2004. We performed CKRS to treat TN in older patients. And a Computed Tomography (CT) cisternography obtained a distortion-free localization of the trigeminal nerve. The treatment target was to designate a $3-5 \mathrm{~mm}$ proximal region's trigeminal nerve, excluding the REZ of the trigeminal nerve in the brain stem. We set treatment planning that the radiation targets could be included in the 80-percent isodose curve with radiosurgery doses of 60-64 Gy. The median follow-up period after CKRS was 36 months (24-48 months). Pain intensity was measured using a Visual Analog Scale (VAS), Quality of Life Scale (QOLS) were measured at baseline and after CKRS. Results: Relief of pain in 28 patients with TN after CKRS showed that pain relief was achieved in 15 patients within the first 24 hours after CKRS. Within 7 days, 25 of the 28 study patients reported early pain relief. The pain control rate was $85.7 \%$ at follow up of 24 months. Pain relapsed in four of 28 patients. Twelve patients had hyperesthesia (35.7\%) with no other complication. The mean ( \pm S.D.) VAS of baseline status was 7.7 $( \pm 0.97)$ before CKRS in patients with TN. VAS decreased to $3.6 \pm 1.8$ within 24 hours after CKRS compared with baseline VAS. The relief of pain was sustained by $2.1 \pm 1.5$ for 1 week and by $1.8 \pm 1.7$ at 6 months. After 12 months and 24 months, VAS remained by $2.0 \pm 1.4$ and 2.1 \pm 1.5 . The dose of gabapentin $(1,200 \pm 600 \mathrm{mg} /$ day $)$ before treatment. The gabapentin dose in the six month did not significantly decrease in taking gabapentin ( $900 \pm 300 \mathrm{mg} /$ day) compared with the baseline dose. Consumption after 24 months was also significantly reduced by $600 \pm 300 \mathrm{mg} /$ day. Conclusion: We conclude that CKRS is a safe and effective treatment for elderly patients with TN in this study. In most patients, the pain was relieved in seven days after CKRS, and no severe complications occurred. However, in older patients, CKRS could be considered as a treatment option for TN.
\end{abstract}

Key Words: CKRS; Cyberknife Radiosurgery; Trigeminal Neuralgia

$\triangle$ Corresponding Author: Tae-Kyu Lee, Department of Neurosurgery, Uijeongbu St. Mary's Hospital, The Catholic University of Korea, College of Medicine, 271, Cheonboro, Uijeongbu 11765, Republic of Korea Tel: +82-31-820-3638, Fax: +82-31-847-8077, E-mail: magpie67@catholic.ac.kr

\section{INTRODUCTION}

Trigeminal Neuralgia (TN) is a neuropathic disorder characterized by episodes of intense pain in the face, originating from the trigeminal nerve ${ }^{4)}$. TN is not easily controlled but can be managed with a variety of treatment options. In curing $\mathrm{TN}$, the medical treatment based on anodynes, anticonvulsants, muscle relaxants. Nerve block based on local anesthetic, alcohol or glycerol on trigeminal neural division and ganglion. Also, RadioFrequency (RF) coagulation and the MicroVascular Decompression (MVD) have been used to treat TN in elderly patients $^{24)}$. CyberKnife (Accuray Inc., Sunnyvale, CA, USA) is constituted by a linear accelerator (LINAC) and a robot arm having six joints ${ }^{1}$. In this study, We retrospectively analyzed the outcome of 28 elderly patients with intractable TN to evaluate whether CKRS is an effective and safe mode of therapy.

\section{MATERIALS AND METHODS}

\section{Subjects}

We collect retrospective data on 28 patients are unsuitable for operation due to old age (over 65 years old) were treated by CKRS with CT cisternography planning since April 1, 2004. Among 28 patients, 8 were male, and 20 were female, ranging between 65 and 86 years old (74.75 \pm 6.35 : Mean \pm SD). Eighteen patients had a location of pain on the right and ten on the left. 
They did not show a response to drugs with NSAIDs, SNRIs and gabapentinoids. Patients were treated with medication for 3 months up to 126 months (12 months: Median). The characteristic of patient groups treated by CKRS is summarized in Table 1. Pain intensity measured using VAS, Quality of life scale (American Chronic Pain Association), and gabapentin consumption were measured at baseline and after CKRS. The patient was scheduled to return to the outpatient clinic after one week during the twenty-four-months follow-up visits after CKRS.

\section{Therapeutic method}

\section{1) Inclusion criteria}

Recently, radiosurgery has been proposed for drug-resistant $\mathrm{TN}$ patients (or in case of relevant drug-related side effects) especially in case of the absence of other primary indications (i.e., neurovascular conflict). RS has to be always considered for patients who are not suitable for surgery and for elderly patients (also in case of MR evidence of neurovascular conflict). Patients' preferences can also be considered. Burchiel's type 1 (or classic) and type 2 are the TN forms most commonly treated by using RS. RS may also be advocated in selected cases of symptomatic (MS-related) and post herpetic TN in some cases of atypical facial pain. The treatment of neurophatic and deafferentation $\mathrm{TN}$ is not generally indicated

\section{2) Procedure}

\section{(1) CT cistemography}

CT cisternography should be performed. Firstly, Isovist of $5 \mathrm{cc}$ should be injected into a thecal sac. Next, the patient should take trendelenburg position for one hour to take an image. The whole head should be scanned at intervals of 1.25 $\mathrm{mm}$ by CT in order that the anatomical shape of basal cistern can be well shown.

\section{(2) Treatment planning}

The target volume was usually defined as a 3- to $5-\mathrm{mm}$ segment of the trigeminal nerve ( $4 \mathrm{~mm}$ in $75 \%$ of cases), localized in the cisternal portion, $3 \mathrm{~mm}$ anterior to the dorsal root entry zone. Patients were treated in a uniformed fashion according to treatment planning that was established to the end that radiation targets could be included in the 80-percent isodose curve with 60-64 Gy radiosurgery doses (Fig. 1).

\section{(3) Follow-up examinations}

After CKRS, patients were evaluated for pain intensity, time to pain relief, presence of facial numbness, or anesthesia dolorosa.
Follow-up assessments were performed every 3 months after CKRS as outpatient visits or by telephone interviews. The first follow-up examination took place at 3 months, and the last available follow-up was referred to as the long-term follow-up. In this study, VAS was used in the pain intensity score system.

\section{Statistical analysis}

Pain intensity measured using VAS, and morphine consumption at baseline and each visit after CKRS. The repeated measure ANOVA is used to determine whether there are any statistically significant differences between the means of baseline and the means of a periodic visit.

\section{RESULT}

\section{Pain relief after CKRS versus baseline}

Relief of pain in 28 patients with TN after CKRS showed that pain relief was achieved in 15 patients within the first 24 hours after treatment. Within 7 days, 25 of the 28 study patients reported early pain relief. The rate of pain control was $85.7 \%$ at follow up of 24months. Pain relapsed in four of 28 patients. Twelve patients had hyperesthesia (35.7\%) with no other complication. The mean ( \pm S.D.) VAS of baseline status was 7.7 $( \pm 0.97)$ before CKRS in patients with TN. VAS decreased to $3.6 \pm 1.8$ within 24 hours after CKRS compared with baseline VAS. The relief of pain was sustained by $2.1 \pm 1.5$ for 1 week and by $1.8 \pm 1.7$ at 6 months. After 12 months and 24 months, VAS remained by $2.0 \pm 1.4$ and $2.1 \pm 1.5$. The repeated measure

Table 1. Demographic characteristics of patients

\begin{tabular}{lc}
\hline Patient characteristics $(\mathrm{n}=28)$ & \\
Age (year) & $65-86$ \\
Range & $74.75 \pm 6.35$ \\
Mean \pm SD & \\
Gender & 13 \\
Female & 10 \\
Male & \\
Location & 16 \\
Right & 7 \\
Left & 8 \\
Lesion & 1 \\
V1 & 8 \\
V2 & 9 \\
V3 & 10 \\
V2+3 & \\
Duration of medication (months) & $24-53$ \\
Range & 38 \\
Median &
\end{tabular}


ANOVA was used to determine whether there was any statistically significant difference between the means of baseline and regular visits. Mean difference (4.6 \pm 0.83$)$ after treatment: $5.6 \pm$ 0.43 after 1 week: $5.8 \pm 0.63$ after 6 month: $5.7 \pm 0.43$ after 12 months: $5.6 \pm 0.43$ after 24 months) between the baseline VAS (7.7 \pm 0.97$)$ and VAS after CKRS is statistically significant (p $<0.001$ ) during follow-up visit for 24 months (Table 2, Fig. 2).

\section{Gabapentin consumption after CKRS versus baseline}

Relief of pain after CKRS lasts for 24 months, which in most cases, for over 6 month. CKRS made it possible to reduce gabapentin consumption. The gabapentin dose in the 6 month significantly decrease in taking gabapentin $(600 \pm 300 \mathrm{mg} /$ day $)$ compared with the dose of gabapentin $(1,200 \pm 600 \mathrm{mg} /$ day $)$ before treatment. Consumption of gabapentin after 24 months was also significantly reduced by $600 \pm 300 \mathrm{mg} /$ day and the baseline doses were $1,200 \pm 600 \mathrm{mg} /$ day that significantly reduced to 900 $\pm 300 \mathrm{mg}$ /day after 7 days after CKRS $(\mathrm{p}<0.001)$. The reduction by $600 \pm 300 \mathrm{mg} /$ day in daily gabapentin consumption was observed at 6 months after CKRS compared with the baseline (Table $3)$. The mean dose value ( \pm S.D.) after 6 months was also significantly reduced by $900 \pm 300 \mathrm{mg} /$ day.

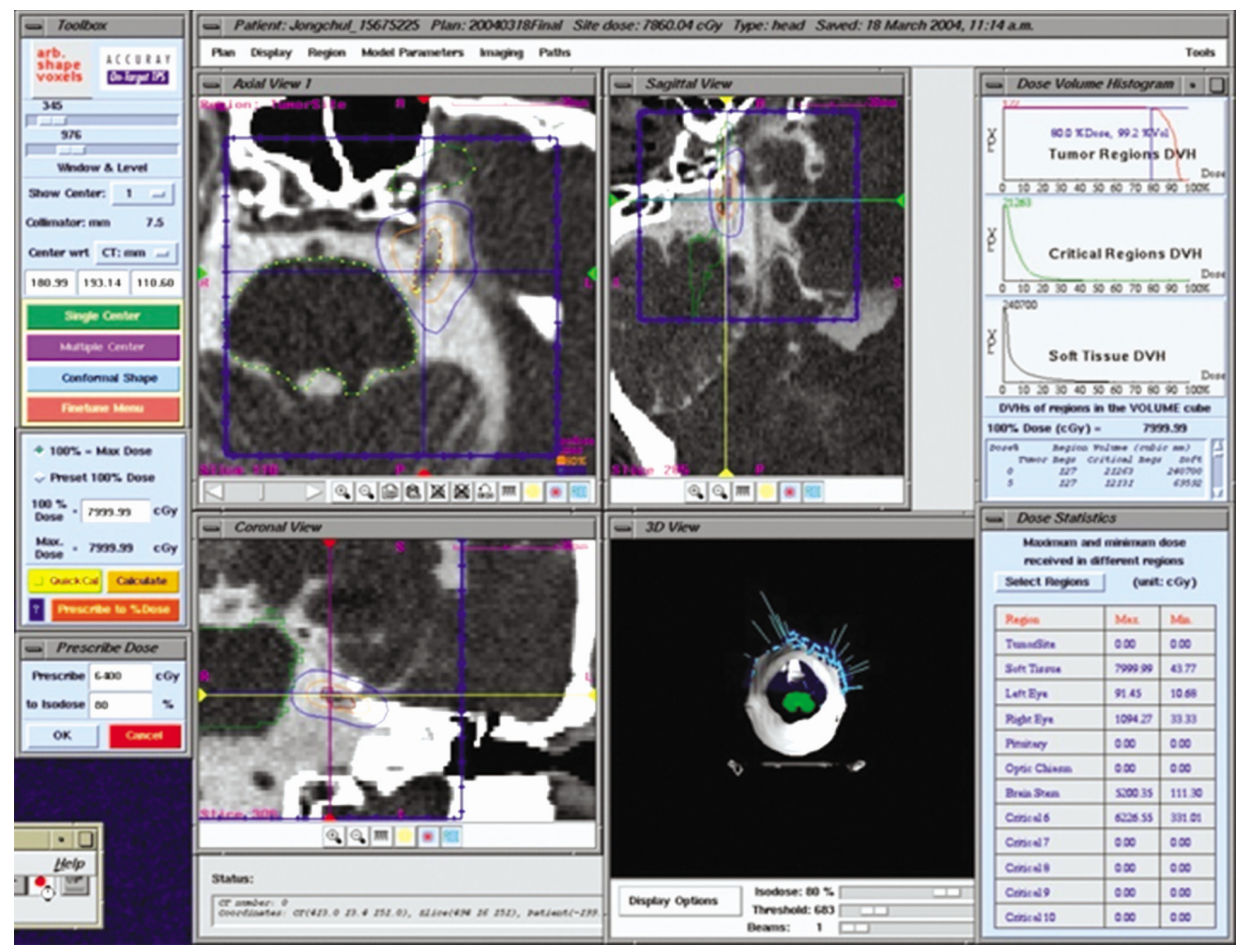

Fig. 1. A CT cisternography was performed to obtain an distortion-free localization of the trigeminal nerve. Treatment planning was established to the end that radiation targets could be all included in the 80-percent isodose curve with radiosurgery doses of 60-64 Gy. The $80 \%$ isodose line was prescribed in a conformal fashion to a 3 to $5-\mathrm{mm}$ length of the nerve, the most proximal point of the nerve identified for the purposes of treatment planning was $3 \mathrm{~mm}$ away from the brainstem.

Table 2. Clinical characteristics stratified by VAS after CKRS

\begin{tabular}{|c|c|c|c|c|c|c|c|}
\hline After CKRS & Baseline Mean & $\begin{array}{l}\text { Treatment } \\
\text { Mean }\end{array}$ & $\begin{array}{c}\text { Mean } \\
\text { Difference }\end{array}$ & $\begin{array}{l}\text { Standard } \\
\text { Deviation }\end{array}$ & $\begin{array}{c}95.00 \% \mathrm{CI} \\
\text { of difference }\end{array}$ & Significant & $\begin{array}{l}\text { Adjusted } \\
p \text {-value }\end{array}$ \\
\hline vs. 24 hours & 7.7 & 3.6 & 4.1 & 1.8 & 2.9 to 4.3 & Yes & 0.001 \\
\hline vs. 1 week & 7.7 & 2.1 & 5.6 & 1.5 & 1.5 to 2.6 & Yes & 0.001 \\
\hline vs. 6 months & 7.7 & 1.8 & 5.8 & 1.7 & 1.1 to 2.4 & Yes & 0.001 \\
\hline vs. 12 months & 7.7 & 2.0 & 5.7 & 1.4 & 1.5 to 2.5 & Yes & 0.001 \\
\hline vs. 24 months & 7.7 & 2.1 & 5.6 & 1.4 & 1.5 to 2.5 & Yes & 0.001 \\
\hline
\end{tabular}



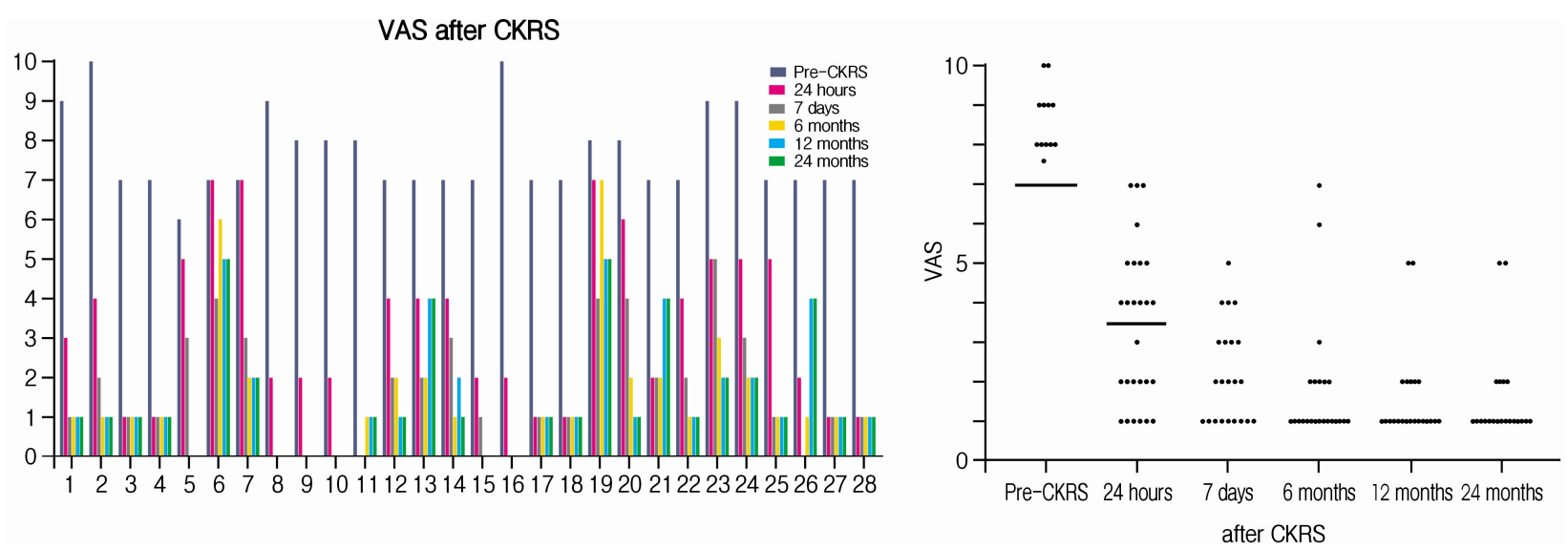

Fig. 2. Clinical Characteristics Stratified by VAS after CKRS.

Table 3. Gabapentin consumption after CKRS

\begin{tabular}{lcc}
\hline \hline Baseline & $\begin{array}{c}\text { Daily Gabapentin } \\
\text { dose }(\mathrm{mg} / \text { day })\end{array}$ & $\begin{array}{c}\text { Pain } \\
\text { Intensity }\end{array}$ \\
\hline 24 Hours after CKRS & $900 \pm 300$ & $3.7[7-10]$ \\
1 week after CKRS & $900 \pm 300$ & $2.1[0-5]$ \\
6 months after CKRS & $600 \pm 300$ & $1.8[0-7]$ \\
12 months after CKRS & $600 \pm 300$ & $2.0[0-5]$ \\
24 months after CKRS & $300 \pm 300$ & $2.1[0-5]$ \\
\hline
\end{tabular}

Daily Gabapentin doses are mean $\pm \mathrm{SD}(\mathrm{mg} /$ day)

Pain intensity is mean value [minimummaximum] in VAS

\section{Side effect}

Hypesthesia was observed in 12 out of 28 patients. The patients with hypesthesia were divided into two main groups: $64 \mathrm{~Gy}$ to the $80 \%$ isodose line was delivered to one group of 9 patients and 60 Gy to the $80 \%$ isodose line was done to another group of 3 patients. This study reported a 35.7\% increase of new hypesthesia symptoms in the follow up period, we observed mild to moderate numbness in the patients. The median time to the onset of numbness was 6 months (range, 2-16 months). Facial hypesthesia was observed in $12(35.7 \%)$ out of 28 patients but all showed excellent or good response at 7 days after CKRS. However, other nervous complications were not observed. Table 2 shows restorative effects, therapeutic dose and adverse reactions.

\section{DISCUSSION}

TN is caused by compression of the trigeminal nerve root by blood vessels, usually within a few millimeters of the junction between the trigeminal nerve root and the pons ${ }^{4,6}$. Dandy initially mentioned the theory of neurovascular compression in
1934. He reported that $66(30.5 \%)$ out of 215 patients, who underwent surgeries through craniotomy, experienced perstriction toward the trigeminal nerve. Likewise, Jannetta advanced his theory for the better. The real frequency of the perstriction towards the trigeminal nerve, observed by occipital craniotomy, ran up from $11 \%$ to $96 \%$. He reported that He should set the trigeminal neuralgia's neurovascular etiology and the penetrative way of the trigeminal nerve root to the therapeutic target ${ }^{24)}$.

The application of RS to treat TN dates back to1950s with Leksell, who first used stereotactic radiosurgerytargeting the gasserian ganglion in humans with encouraging results ${ }^{10)}$. Nowadays, the RS technique is a well-established option for the treatment of TN, and the series reported confirm the efficacy and safety of this modality Northwest Neuroscience Institute's Young et al. and Pollock et al., who have reported multi-institutional researches, dealt with safety and efficacy of radiosurgery in detail among their reports ${ }^{3,15,16,21,23)}$. The same as this study, such reports claimed that the penetrative way of trigeminal nerve root is the target of $\mathrm{RS}^{2,9,14,23)}$.

The rationale about the trigeminal neuralgia relief after RS is related to the effect on nervous amyelinic, low caliber cells associated to nociceptive sensibility, and to the destruction of the ionic channels and relative blockade of the electrical conduction $^{12,22)}$. Hodaie et al. by using tractography has helped the neurologiocal community reach a better understanding of the effect of RS on the trigeminal nerve. The authors have observed that radial but not axial diffusivities changed markedly after RS, suggesting that this irradiation technique primarily affects myelin. Also, tractography seems to be a more sensitive imaging tool than conventional gadolinium-enhanced MR, as confirmed by the fact that, for patients with long-term follow-up, recovery of $\mathrm{FA} / \mathrm{RD}$ correlated with pain recurrence ${ }^{5}$.

The claim is based on the experiences achieved through microneurosurgery. We observed initial pain relief in $89.5 \%$ (24 out of 28) of the patients with TN. Our results are consistent 
with the reported rates from studies of gamma knife (Elekta $\mathrm{AB}$, Stockholm, Sweden) and linear accelerator (LINAC) radiosurgery, which have reported initial pain relief of 50 to $96 \%$. This study is the result derived from applying CKRS to treat TN. After CKRS, the pain relief was analyzed on the basis of VAS system. 28 patients showed excellent response within 7 days. The reason why their pain were alleviated so rapidly can be explained by several causes. First, its accuracy is higher than the existing instruments because the therapeutic target is set by using positive contrast CT cisternography. Second, treatment planning and irradiation can be performed without any restrictions because there is no stereotactic ring. Lastly, isodose distribution can be easily equalized along the trigeminal nerve $\mathrm{e}^{2,11,18)}$. At periods of 2 years follow-up, $68 \%$ of our patients continued to have excellent pain relief. This is also comparable to other modes of radiosurgery. At gamma knife centers, 40 to 59\% sustained pain relief was observed at 2 to 5 years follow-up ${ }^{3,12,15,20,22)}$.

Hypoesthesia/dysesthesia is the most common adverse event. The median hypoesthesia rate ranges between $19 \%$ and $28 \%$. Others toxicities including anesthesia dolorosa, dysgeusia, dry eye syndrome, hearing impairment, and masticator weakness are very $\operatorname{rare}^{8,11,13,14,17,18,21)}$. Factors which may affect the toxicity are the target selection, the length of the irradiated nerve, and the dose. Some studies seem to suggest a potential advantage of the intracisternal target compared to the REZ in terms of complication rate. Some other authors found that longer portions of irradiated nerve as well as doses higher than 90 Gy may lead to a higher toxicity rate. This study reported a $32 \%$ increase of new hypesthesia symptoms in the follow-up period, which we observed mild to moderate numbness in the patients. The median time to the onset of numbness was 10 months (range, 2-16 months) $)^{22)}$.

The merit of CKRS is that homogeneous conformity can be formed, the ray can be accurately irradiated and the therapeutic target can be accurately set ${ }^{1)}$. Specifically, it is impossible to get satisfactory dose distribution from the thin and long trigeminal nerve by performing one-time spherical irradiation ${ }^{7)}$. Dose distribution can be satisfiably formed though repetitive irradiation based on secondary isocenter, but it increases morbidity $^{11,18)}$. The accuracy of CKRS is based on the accuracy of ray emitting and target positioning. The accuracy of ray emitting can be high by the robot arm with six joints and the image-seeking camera system. The accuracy of target positioning can be high by the mutual function between CT imaging and treatment planning ${ }^{18,19)}$. According to a phantom research on CKRS, the collimation error scored $1.1 \pm 0.3 \mathrm{~mm}$ when CTscanning intervals were $1.25 \mathrm{~mm}$. The error emarkably improved in comparison with the collimation error of $5 \mathrm{~mm}$, derived from the treatment planning of conformal radiosurgery on the basis of the data gotten by MRI. It is also reasonable to speculate that the short latency and excellent response obtained in this series stems from better treatment accuracy ${ }^{1,7)}$. CKRS with CT cisternography may have enhanced overall accuracy, which in turn may have been manifested in greater treatment efficacy $^{18,19)}$.

\section{CONCLUSION}

In conclusion, this clinical study suggests that CKRS is a safe and effective treatment for Elderly Patients with TN in this study. In most patients, the pain was relieved in seven days after CKRS, and no severe complications occurred. Howe- ver, In older patients, CKRS could be considered as a treatment option for TN.

\section{REFERENCES}

1. Adler Jr JR, Chang SD, Murphy MJ, Doty J, Geis P, Hancock SL: The Cyberknife: A frameless robotic system for radiosurgery. Stereotactic and Functional Neurosurgery 69:124-128, 1997

2. Chan MD, Shaw EG, Tatter SB: Radiosurgical management of trigeminal neuralgia. Neurosurg Clin N Am 24:613-621, 2013

3. Conti A, Acker G, Pontoriero A, Hardt J, Kluge A, Cacciola A, et al.: Factors affecting outcome in frameless non-isocentric stereotactic radiosurgery for trigeminal neuralgia: A multicentric cohort study. Radiation Oncology 15:1-10, 2020

4. Dandy WE: Concerning the cause of trigeminal neuralgia. The American Journal of Surgery 24:447-455, 1934

5. Hodaie M, Chen DQ, Quan J, Laperriere N: Tractography delineates microstructural changes in the trigeminal nerve after focal radiosurgery for trigeminal neuralgia. PLoS One 7:e32745, 2012

6. Jannetta PJ: Arterial compression of the trigeminal nerve at the pons in patients with trigeminal neuralgia. Journal of Neurosurgery 26:159-162, 1967

7. Kilby W, Dooley J, Kuduvalli G, Sayeh S, Maurer Jr C: The CyberKnife ${ }^{\circledR}$ robotic radiosurgery system in 2010 . Technology in Cancer Research \& Treatment 9:433-452, 2010

8. Kondziolka D, Lunsford LD, Flickinger JC, Young RF, Vermeulen S, Duma CM, et al.: Stereotactic radiosurgery for trigeminal neuralgia: A multiinstitutional study using the gamma unit. Journal of Neurosurgery 84:940-945, 1996

9. Kondziolka D, Zorro O, Lobato-Polo J, Kano H, Flannery TJ, Flickinger JC, et al.: Gamma Knife stereotactic radiosurgery for idiopathic trigeminal neuralgia clinical article. Journal of Neurosurgery 112:758-765, 2010

10. Larsson B, Leksell L, Rexed B, Sourander P, Mair W, Andersson $\mathrm{B}$ : The high-energy proton beam as a neurosurgical tool. Nature 182:1222-1223, 1958

11. Lim M, Villavicencio AT, Burneikiene S, Chang SD, Romanelli P, McNeely L, et al.: CyberKnife radiosurgery for idiopathic trigeminal neuralgia. Neurosurgical Focus 18:1-7, 2005

12. Marchetti M, Pinzi V, De Martin E, Ghielmetti F, Fariselli L: Radiosurgery for trigeminal neuralgia: the state of art. Neurological Sciences 40:153-157, 2019

13. Pan HC, Sheehan J, Huang CF, Sheu ML, Yang DY, Chiu WT: Quality-of-life outcomes after Gamma Knife surgery for trigemi- 
nal neuralgia. J Neurosurg 113 Suppl:191-198, 2010

14. Pollock BE, Phuong LK, Gorman DA, Foote RL, Stafford SL: Stereotactic radiosurgery for idiopathic trigeminal neuralgia. Journal of Neurosurgery 97:347-353, 2002

15. Raygor KP, Lee AT, Nichols N, Wang DD, Ward MM, Barbaro $\mathrm{NM}$, et al.: Long-term pain outcomes in elderly patients with trigeminal neuralgia: comparison of first-time microvascular decompression and stereotactic radiosurgery. Neurosurgical Focus 49: E23, 2020

16. Regis J, Tuleasca C: Fifteen years of Gamma Knife surgery for trigeminal neuralgia in the Journal of Neurosurgery: History of a revolution in functional neurosurgery. J Neurosurg 115 Suppl: 2-7, 2011

17. Riesenburger RI, Hwang SW, Schirmer CM, Zerris V, Wu JK, Mahn K, et al.: Outcomes following single-treatment Gamma Knife surgery for trigeminal neuralgia with a minimum 3-year follow-up. J Neurosurg 112:766-771, 2010

18. Romanelli P, Heit G, Chang SD, Martin D, Pham C, Adler J: Cyberknife radiosurgery for trigeminal neuralgia. Stereotactic and Functional Neurosurgery 81:105-109, 2004

19. Romanelli P, Conti A, Bianchi L, Bergantin A, Martinotti A, Belt- ramo G: Image-guided robotic radiosurgery for trigeminal neuralgia. Neurosurgery 83:1023-1030, 2018

20. Tuleasca C, Régis J, Sahgal A, De Salles A, Hayashi M, Ma L, et al.: Stereotactic radiosurgery for trigeminal neuralgia: A systematic review: International Stereotactic Radiosurgery Society Practice Guidelines. Journal of Neurosurgery 130:733-757, 2018

21. Weller M, Marshall K, Lovato JF, Bourland JD, deGuzman AF, Munley MT, et al.: Single-institution retrospective series of Gamma Knife radiosurgery in the treatment of multiple sclerosis related trigeminal neuralgia: Factors that predict efficacy. Stereotact Funct Neurosurg 92:53-58, 2014

22. Wilson TA, Karlsson B, Huang L, Ramanathan D, Oyoyo U, Boling W: Optimizing radiosurgery for trigeminal neuralgia: Impact of radiation dose and anatomic target on patient outcomes. World Neurosurgery, 2020

23. Young B, Shivazad A, Kryscio RJ, St Clair W, Bush HM: Longterm outcome of high-dose gamma knife surgery in treatment of trigeminal neuralgia. J Neurosurg 119:1166-1175, 2013

24. Zakrzewska JM, Linskey ME: Trigeminal neuralgia. BMJ 348: g474, 2014 